\title{
COVID-19 PRESENTING WITH CENTRAL RETINAL VEIN OCCLUSION: A CASE REPORT
}

\section{Kalina Z. TRIFONOVA ${ }^{1}$, Kiril S. SLAVEYKOV ${ }^{1 凶}$, Ivailo S. DOCHEV ${ }^{1}$, Pavlina 0. PARUSHEVA², Liliya M. PEKOVA ${ }^{2}$}

${ }^{1}$ Department of Ophthalmology, Trakia University, Medical Faculty, Stara Zagora, Bulgaria

${ }^{2}$ Department of Infectious diseases, Trakia University, Medical Faculty, Stara Zagora, Bulgaria

Received 26 April 2021, Accepted 19 May 2021

https://doi.org/10.31688/ABMU.2021.56.2.19

\section{Abstract}

Introduction. Ophthalmologists worldwide are reporting various ophthalmic manifestations of coronavirus disease 2019 (COVID-19). The most common ocular manifestation is conjunctivitis. Even a rarer complication, retinal vein occlusion may appear in patients with COVID-19.

Case presentation. A 52-year-old man was admitted for retinal vein occlusion of the right eye. $\mathrm{He}$ had been complaining of blurred vision for several days. A complete clinical and paraclinical evaluation of the patient was performed, in search of associated diseases. The patient tested positive for infection with severe acute respiratory syndrome coronavirus 2 (SARS-CoV-2).

Conclusions. Retinal circulation is a potential site for thromboembolic complications in patients with COVID-19. Ophthalmologists should be aware of this possible association and maintain a high level of suspicion of COVID-19 in patients with vascular occlusive diseases of the eye.

Keywords: thromboembolism, ocular complication, SARS-CoV-2.

\section{Résumé}

COVID-19 présentant avec une occlusion de la veine rétinienne centrale

Introduction. Les ophtalmologistes du monde entier signalent diverses manifestations ophtalmiques de la maladie à coronavirus 2019 (COVID-19). La manifestation oculaire la plus fréquente est la conjonctivite. Une complication encore plus rare, l'occlusion de la veine rétinienne, peut apparaitre chez les patients atteints de COVID-19.

Présentation du cas. Un homme de 52 ans a été admis pour l'occlusion veineuse rétinienne de l'œil droit. Il se plaignait d'une vision trouble depuis plusieurs jours. Une évaluation clinique et paraclinique complète du patient a été réalisée, à la recherche de pathologies associées. Le patient a été testé positif à l'infection par le coronavirus 2 du syndrome respiratoire aigu sévère (SARS-CoV-2).

Conclusions. La circulation rétinienne est un site potentiel de complications thromboemboliques chez les patients atteints de COVID-19. Les ophtalmologistes doivent être conscients de cette association possible et maintenir un niveau élevé de suspicion de COVID-19 


\section{List of abbreviations:}

COVID-19 - coronavirus disease 2019

SARS-CoV-2 - severe acute respiratory syndrome coronavirus 2

AST - aspartate aminotransferase

CRP - C-reactive protein

RT-PCR - real-time polymerase chain reaction

\section{INTRODUCTION}

Coronavirus disease 2019 (COVID-19) may pose challenges regarding the clinical diagnosis, as there is no pathognomonic symptom of the disease. Several clinical symptoms have been frequently reported among COVID-19 patients, including, but not limited to, cough, fever, fatigue, sore throat, nasal obstruction, shortness of breath, headache, sputum production, and hemoptysis ${ }^{1}$. However, the disease can affect almost every organ of the body. The visual system is no exception. Ophthalmologists worldwide are reporting various ocular manifestations of the disease. The most common ocular manifestation is conjunctivitis, that can be the first and sometimes the only sign of the disease ${ }^{2}$. Other rarely reported manifestations are episcleritis ${ }^{2}$, optic neuritis, cranial neuropathies, Miller-Fishes syndrome, anterior ischemic optic neuropathy, retinal artery and retinal vein occlusions, papillophlebitis and other retinal microvascular changes ${ }^{3}$.

\section{Case presentation}

We present the case of a 52-year-old man from Tulovo, Bulgaria, who visited an outpatient clinic complaining of blurred vision of his right eye. The chez les patients atteints de maladies vasculaires occlusives de l'œil.

Mots-clés: thromboembolie, complication oculaire, SARS-CoV-2.

patient was diagnosed with central retinal vein occlusion. He refused hospitalization and was prescribed pentoxyphilline SR $400 \mathrm{mg}$ two times per day. Two days later, the patient was admitted in the Ophthalmology Department of the University Hospital Stara Zagora, Bulgaria. His best-corrected visual acuity was 20/80 in the right eye and 20/20 in the left eye on Snellen's chart. The intraocular pressure was $16 \mathrm{mmHg}$ in right eye and $17 \mathrm{mmHg}$ in left eye. The pupil of his right eye had mild afferent pupillary defect. The anterior segment of both eyes was unremarkable. A dilated-pupil fundus examination was performed with indirect ophthalmoscopy. There was disk swelling, with flame-shaped hemorrhages around the optic disk in the right eye. The veins were dilated and tortuous, with rare scattered dot and blot and flame-shaped hemorrhages throughout the whole retina. There were no changes in the fundus of the left eye. Since the patient was relatively young, with no predisposing risk factors for developing this complication, a general check-up was decided. The patient denied any pathological history. The body mass index and blood pressure values were normal. The results of the blood tests revealed only lymphopenia, and elevated aspartate aminotransferase (AST) (Table 1).

Table 1. The results of paraclinical investigations performed in the Department of Ophthalmology at admission.

\begin{tabular}{ccc}
\hline Test & Level & Normal range \\
\hline Serum glucose $(\mathrm{mmol} / \mathrm{L})$ & 5.63 & $2.8-5.6$ \\
\hline Cholesterol $(\mathrm{mmol} / \mathrm{L})$ & 4.51 & $3.4-5.2$ \\
\hline Triglycerides $(\mathrm{mmol} / \mathrm{L})$ & 1.34 & $0.6-2.2$ \\
\hline AST $(\mathrm{IU} / \mathrm{L})$ & 61.4 & $0-37$ \\
\hline Creatinine $($ micromoles/L) & 88 & $80-127$ \\
\hline White blood cells & $4.210^{\wedge} 9 / \mathrm{L}$ & $3.5-10.5$ \\
\hline Lymphocytes & $0.5710^{\wedge} 9 / \mathrm{L}$ & $0.8-4.2$ \\
\hline Granulocytes & $3.2610^{\wedge} 9 / \mathrm{L}$ & $2-7.9$ \\
\hline Erythrocytes & $5.7210^{\wedge} 12 / \mathrm{L}$ & $4.5-5.8$ \\
\hline Hemoglobin $(\mathrm{g} / \mathrm{L})$ & 159 & $135-180$ \\
\hline Hematocrit $(\%)$ & 45.6 & $40-54$ \\
\hline Thrombocytes & $14010 \wedge 9 / \mathrm{L}$ & $140-440$ \\
\hline Prothrombin time $(\mathrm{s})$ & 13.4 & $11-15$ \\
\hline INR & 1.16 & $0.9-1.3$ \\
\hline
\end{tabular}


On the next day, the patient felt unwell, with chills, and lack of appetite. There were no upper respiratory symptoms. The body temperature was normal $\left(36.4^{\circ} \mathrm{C}\right)$. The C-reactive protein (CRP) level was moderately elevated. A rapid antigen test for COVID-19 was done, with positive result. Real-time polymerase chain reaction (RT-PCR) test from a nasopharyngeal swab was also positive, the patient being transferred to the Department of Infectious Diseases.

Fundus changes were documented with Welch-Allyn iExaminer, to avoid transportation

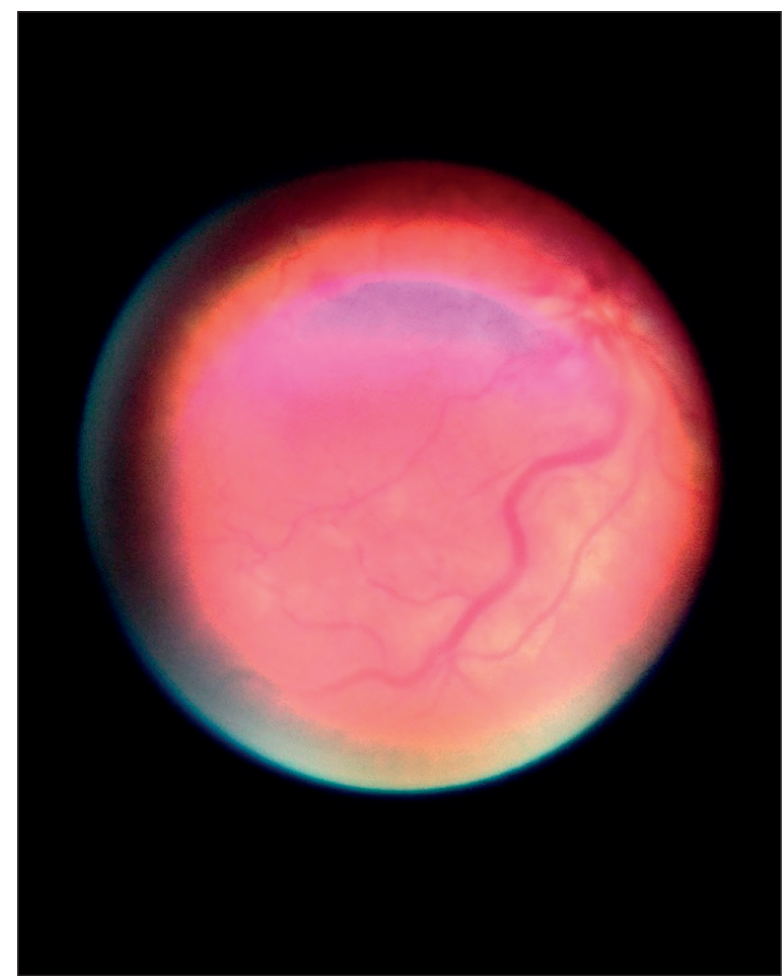

Figure 1. Welch-Allyn IExaminer fundus photography of the right eye - disk swelling with flame shaped hemorrhages around the optic disk, dilated and tortuous veins, rare scattered dot and blot and flame shaped hemorrhag

Table 2. The results of paraclinical investigations performed in the Department of Infectious Diseases

\begin{tabular}{ccc}
\hline Marker & Level & Normal range \\
\hline Date & $3^{\text {rd }}$ day & \\
\hline Fibrinogen $(\mathrm{g} / \mathrm{L})$ & 3.44 & $2-4.0$ \\
\hline $\begin{array}{c}\text { D-Dimers }(\mu \mathrm{g} / \\
\mathrm{mL})\end{array}$ & 8.99 & $0-0.5$ \\
\hline Date & $5^{\text {th }}$ day & \\
\hline LDH & $455 \mathrm{IU} / \mathrm{L}$ & $225-450$ \\
\hline $\begin{array}{c}\text { D-Dimers }(\mu \mathrm{g} / \\
\mathrm{mL})\end{array}$ & 0.63 & $0-0.5$ \\
\hline Date & $7^{\text {th }}$ day & \\
\hline CRP & 15 & $0-6$ \\
\hline
\end{tabular}

of the patient and contamination of other devices (Fig. 1). The chest X-ray showed characteristic imaging findings of SARS-CoV-2 pneumonia, with multiple bilateral ground glass opacities (Fig. 2). The oxygen saturation was $91 \%$ in aa, and supplemental oxygen therapy was started. Additional blood tests were performed, which showed normal fibrinogen, but very high D-Dimers $(8.99 \mu \mathrm{g} / \mathrm{mL})$ (Table 2). Treatment with systemic corticosteroids, low molecular weight heparin and intravenous antibiotic was started (Table 3). Isoprinosine, vitamin C 1000 and vitamin D3 were also added, as adjunctive therapy.

The patient announced improvement in his vision on the next day. After five days of treatment, D-Dimers were normal $(0.63 \mu \mathrm{g} / \mathrm{mL})$, and after seven days the CRP decreased. The patient was discharged in a stable state, with normal oxygen saturation and almost normal vision. According to the law in Bulgaria, he had to stay at home under a quarantine for additional 14 days. The patient have not presented for follow-up.

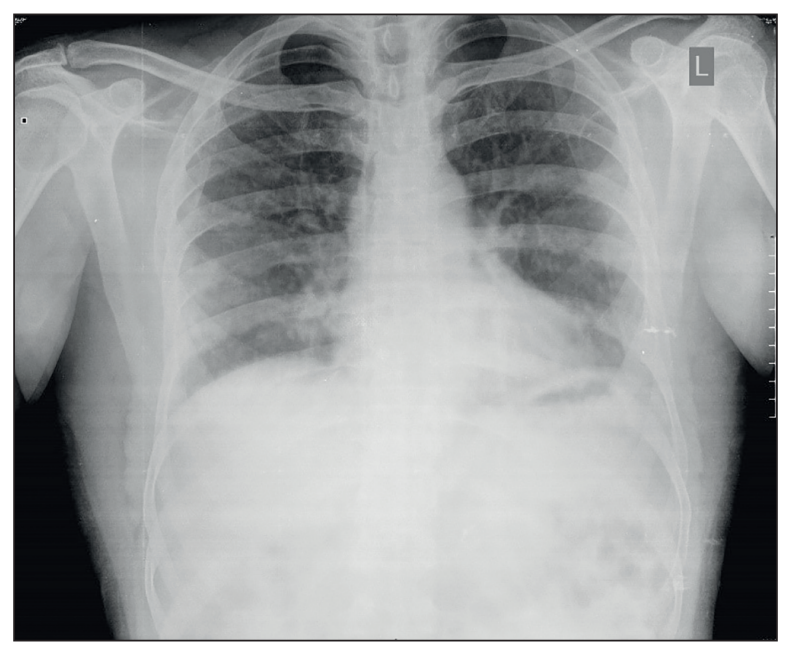

Figure 2. A chest $X$ ray - characteristic changes in the lungs for COVID-19 with multiple bilateral ground glass opacities

Table 3. Drug treatment in the Department of Infectious Diseases

\begin{tabular}{cc}
\hline Treatment & Dosage \\
\hline Methylprednisolone & 40 mg b.i.d. \\
\hline Heparin & 2,500 Units t.i.d. \\
\hline Ceftriaxone & $2 \mathrm{~g}$ b.i.d. \\
\hline Isoprinosine & 1,0 g t.i.d. \\
\hline Vitamin C & 1000 mg q.d. \\
\hline Vitamin D3 & 1000 E q.d. \\
\hline Probiotic & 1 capsule q.d.
\end{tabular}




\section{Discussion}

Retinal vein occlusion is most commonly associated with advanced age and arterial hypertension. A patient diagnosed with retinal vein occlusion should have a cardiovascular examination, to detect the etiology. Patients with COVID-19 seem to have a higher risk of retinal vein occlusion ${ }^{47}$. Cases of retinal vein occlusions developing after a recent infection with SARS-CoV-2 have been described ${ }^{4-7}$. Sometimes, COVID-19 and retinal vein occlusion appear in the same time $e^{8-11}$.

The exact mechanism of the development of retinal vascular occlusive diseases in patients with COVID-19 is not clear yet. Central retinal vein occlusion is commonly seen in association with a hypercoagulable state, which may contribute to the pathogen$\mathrm{esis}^{12}$. Infection with SARS-CoV-2 is known to induce a hypercoagulable state, with increased risk of venous thromboembolism ${ }^{13}$. In hospitalized patients with COVID-19, lab abnormalities may include modest prolongation of prothrombin times in about $5 \%$ of cases, mild thrombocytopenia in approximately $30 \%$ of cases, and elevated D-dimers values in $43-60 \%$ of cases $^{14}$.

Some authors explain the higher risk of venous thromboembolism by the endothelial lesions caused by systemic inflammation. Post-mortem analysis show evidence of direct viral infection of the endothelial cells and diffuse endothelial inflammation, leading to endothelial dysfunction and a procoagulant state ${ }^{15}$. Sheth et al. present the case of a 52 -year-old patient, who developed retinal vein occlusion 10 days after testing positive for COVID-19? . The authors support the mechanism of thrombo-inflammatory state secondary to the "cytokine storm", caused by type-3 hypersensitivity (immune-complex disease) rather than direct involvement of viral particles. In support of this theory, the visual acuity of our patient improved with systemic corticosteroid therapy. Similarly, another case of a 54-year-old patient, with impending retinal vein occlusion and COVID-19 pneumonia, treated with systemic corticosteroids, had a complete resolution of the occlusion ${ }^{9}$. On the contrary, Reddy et al..$^{16} \mathrm{com}$ mented that COVID-associated vasculopathy needs to be distinguished from vasculitis. Duff et al. ${ }^{11}$ also support the idea that the retinal vein occlusion that they described in a 74-year-old patient is a thromboembolic event rather than retinal vasculitis, since other findings of retinal vasculitis were not evident at fluorescein angiography three months after the initial vein occlusion. However, these authors still consider that retinal vasculitis, rather than a thrombotic event, should be considered in a COVID-19 patient with ocular changes ${ }^{11}$.

Clinicians should be aware of the risk of vascular occlusive events in patients with COVID-19. Visual symptoms may occur even in mild forms of the disease. A high suspicion for acute ischemia and thrombosis in the proper clinical scenario must be maintained, as delaying the intervention can result in irreversible destruction of vital tissues ${ }^{17}$. Retinal vein occlusion in an otherwise healthy, young patient, may be a potential manifestation of the thrombo-inflammatory state associated with SARS-CoV-2 infection ${ }^{6}$ and may help distinguish COVID-19 from other common forms of upper respiratory illness.

Ophthalmologists need to be very careful while examining patients during the COVID-19 pandemic, because the ocular examination techniques involve a very close contact with the patient ${ }^{18}$. Although SARS-CoV-2 has been detected in the conjunctival secretions or tears of patients with COVID-19 and conjunctivitis, the transmission of infection through respiratory droplets to ophthalmologists without eye protection or masks may be a big concern. During the COVID-19 pandemic, the use of indirect ophthalmoscopy may be a safer option.

Ceran and Ozates stated that older age, high fever, increased neutrophil/lymphocyte ratio, and high levels of acute phase reactants seemed to be risk factors for ocular involvement ${ }^{19}$. Wu et al. reported that ocular involvement in COVID-19 is more likely to be associated with severe pneumonia, compared with the presentation of patients with milder symptoms and normal test results ${ }^{20}$.

\section{Conclusions}

Retinal circulation should be considered as a potential site for thromboembolic complications in COVID-19 patients. The knowledge of this possible association can be useful for an accurate diagnosis. The ophthalmologists should evaluate patients with caution of possible contamination, to further limit the spreading of this highly contagious disease. Vasculitic pathophysiology should not be excluded when deciding the treatment of retinal vascular occlusive disease. Further investigations of the relation between the severity of COVID-19 and ocular complications need to be done.

\section{Authors' Contributions:}

Conceptualization, K.Z.T. and K.S.S, diagnostic procedures, clinical diagnosis - K.S.S, K.Z.T., treatment decisions - L.M.P, P.O.P, analysis, interpretation - K.Z.T, I.S.D, writing - original draft preparation, K.Z.T, K.S.S, I.S.D; writing - review and editing, K.S.S, L.M.P.

\section{Compliance with Ethics Requirements:}

"The authors declare no conflict of interest regarding this article" 
"The authors declare that all the procedures and experiments of this study respect the ethical standards in the Helsinki Declaration of 1975, as revised in 2008(5), as well as the national law. Informed consent was obtained from the patient included in the study"

"No funding for this study"

\section{Acknowledgements:}

None

\section{References}

1. Jin X, Lian JS, Hu JH, et al. Epidemiological, clinical and virological characteristics of 74 cases of coronavirus-infected disease 2019 (COVID-19) with gastrointestinal symptoms. Gut 2020; 69:1002-1009

2. Sen M, Honavar SG, Sharma N, Sachdev MS. COVID-19 and eye: a review of ophthalmic manifestations of COVID-19. Indian Journal of Ophthalmology. 2021;69(3):488-509

3. Tisdale AK, Chwalisz BK. Neuro-ophthalmic manifestations of coronavirus disease 19. Current Opinion in Ophthalmology. 2020;31(6):489-494

4. Yahalomi T, Pikkel J, Arnon R, Pessach Y. Central retinal vein occlusion in a young healthy COVID-19 patient: A case report. American Journal of Ophthalmology Case Reports. 2020;20:100992.

5. Walinjkar J, Makhija S, Sharma H, Morekar S, Natarajan S. Central retinal vein occlusion with COVID-19 infection as the presumptive etiology. Indian Journal of Ophthalmology. 2020;68(11):2572-2574

6. Finn A, Khurana R, Chang L. Hemi-retinal vein occlusion in a young patient with COVID-19. American Journal of Ophthalmology Case Reports. 2021; 22:101046.

7. Sheth J, Narayanan R, Goyal J, Goyal V. Retinal vein occlusion in COVID-19: A novel entity. Indian Journal of Ophthalmology. 2020; 68(10):2291-2293.

8. Kapatayes N, Joondeph B. Retinal vein occlusion associated with COVID-19. Retina Today. 2020;32-33.
9. Invernizzi $\mathrm{A}$, Pellegrini $\mathrm{M}$, Messenio $\mathrm{D}$, et al. Impending central retinal vein occlusion in a patient with coronavirus disease 2019 (COVID-19). Ocular Immunology and Inflammation. 2020;28(8):1290-1292

10. Gaba W, Ahmed D, Al Nuaimi R, Dhanhani A, Eatamadi H. Bilateral central retinal vein occlusion in a 40-year-old man with severe coronavirus disease 2019 (COVID-19) pneumonia. American Journal of Case Reports. 2020; 21:e927691.

11. Duff S, Wilde M, Khurshid G. Branch retinal vein occlusion in a COVID-19 positive patient. The Cureus Journal of Medical Sciences. 2021;13(2):e13586

12. Abu El-Asrar A, Abdel Gader A, Al-Amro S, Al-Momen A. Hypercoagulable states in patients with retinal venous occlusion. Documenta Ophthalmologica. 1998;95(2):133-143

13. Panigada $M$, Bottino $N$, Tagliabue $P$, et al. Hypercoagulability of COVID-19 patients in intensive care unit: A report of thromboelastography findings and other parameters of hemostasis. Journal of Thrombosis Haemostasis. 2020;18(7):1738-1742

14. Tang N, Li D, Wang X, Sun Z. Abnormal coagulation parameters are associated with poor prognosis in patients with novel coronavirus pneumonia. Journal of Thrombosis Haemost asis. 2020;18(4):844-847.

15. Varga Z, Flammer A, Steiger P, et al. Endothelial cell infection and endothelitis in COVID-19. Lancet. 2020;395:14171418.

16. Reddy P, Singh D, Baharani A. Comments on: Retinal vein occlusion in COVID-19: A novel entity. Indian Journal of Ophthalmology. 2021;69(1):192.

17. Hayreh S, Zimmerman M, Kimura A, Sanon A. Central retinal artery occlusion. Retinal survival time. Experimental Eye Research. 2004;78(3):723-736.

18. Kuo I, O'Brien T. COVID-19 and ophthalmology: an underappreciated occupational hazard. Infection Control\& Hospital Epidemiology. 2020;41(10):1207-1208.

19. Ceran B, Ozates S. Ocular manifestations of coronavirus disease 2019. Graefes Archive for Clinical and Experimental Ophthalmology. 2020;258(9):1959-1963.

20. Wu P, Duan F, Luo C, et al. Characteristics of ocular findings of patients with coronavirus disease 2019 (COVID-19) in Hubei Province, China. JAMA Ophthalmology. 2020; 138(5):575-578. 